\title{
ANALYTIC REPRESENTATION OF THE DISTRIBUTIONAL FINITE HANKEL TRANSFORM
}

\author{
O. P. SINGH \\ Uepartment of Mathematics \\ Banaras Hindu University \\ Varanasi 221005, India \\ and \\ RAM S. PATHAK \\ Department of Mathematics \\ Faculty of Science \\ King Saud University \\ Riyadh, Saudi Arabia \\ (Received Apri1 30, 1983)
}

AESTRACT. Various representations of finite Hankel transforris of generalized functions are obtainec. One of the representations is shown to be the limit of a certain iamily of regular generalized functions and this limit is interpreted as a process of truncation for the generalized functions (distributions). An inversion theurem for the serieralized finite Hankel transform is established (in the distributional selise) which gives a Fourier-Bessel series representation of gereral ized functions.

KEY WORDS AND PHRASES. Hankel tranojorms of gereralized furietions, r'est junction. spaces, Firite ilarikel transforms.

1980 MATHEMATICS SUBJECT CLASSIFICATION CODE. 46F12, 44A20, $44 A 15$.

1. INTROCLCTION.

Zemanian [1] extended Hankel transformations to the distribution space $H_{\mu}^{\prime} \cdot H_{\mu}^{\prime}$ is the dual of the space of testing functions $H_{\mu}$ defined as follows: for each real number $\mu$, let $H_{\mu}=\{\phi:(0, \infty) \rightarrow \phi \mid \phi$ is smooth on $(0, \infty)$ and $\phi$ satisfies (1.1) $\}$

$\gamma_{m, k}^{\mu}(\phi)=\sup _{0<x<\infty}\left|x^{m}\left(x^{-1} D\right)^{k}\left[x^{-\mu-\frac{1}{2}} \phi(x)\right]\right|<\infty$, for each $m, k=0,1,2 \ldots$

$H_{\mu}^{\prime}$ consists of certain distributions of slow growth. Then later [2] he obtained a more general result by removing the restriction on the slow growth of the distributions. He defined the Hankel transformation of a distribution of rapid growth in the space $B_{\mu}^{\prime} \cdot B_{\mu}^{\prime}$ is the dual of $B_{\mu}$, the strict inductive 1 imit of the testing 
function spaces $B_{\mu, b}$ (defined in section 2) as $b$ tends to infinity through a monotonically increasing sequence of positive numbers.

We take advantage of the fact that functions in $B_{\mu, b}$ are identically zero after $b$, to define the finite Hankel transformation for the generalized functions in its dual $B_{\mu, b}^{\prime}$. This is done by generalizing Parseval's equation. We find that for $\mu \geq-\frac{1}{2}$, the finite Hankel transform $h_{\mu}$ maps $B_{\mu, b}^{\prime}$ isomorphically onto the generalized function space $Y_{\mu, b}^{\prime}$ (defined in section 3 ). The aim of the present paper is to obtain various representations of the generalized functions in $Y_{\mu, b}$ and to find an inversion formula for the generalized finite Hanke! transform which also gives annther representation of the members of $B_{\mu, b}^{\prime}$ as a Fourier-Bessel series.

We follow the notation and terminology of Schwartz [3] and Zemanian [4,5]. Here I dellotes the open interval $(0, \infty)$. The letters $x, y, t$ and $w$ are used as real variables on 1 . The $k^{\text {th }}$ derivative of an ordinary or generalized function $f(x)$ is usualiy denoted by ${\sigma^{k}}^{k}(x)$ (trough the symbol $D_{x}^{k} f(x)$ is also used). $[(1)$ denotes the space of smooth furctions that have compact support or I. The topology of $D(I)$ is that which makes its dual the space $D^{\prime}(I)$ of Scriwartz's distributicis $[\hat{2}$, vol. I, p.65].

2. TESTING FUNCTION SPACES $\mathrm{B}_{\mu, b}$ AND $Y_{\mu, b}$.

Let $b>0$ be a fixeci arbitrary real number. Then for $\mu \in R$, where $R$ is the net of rea? numbers, we define

$$
\begin{aligned}
B_{\mu, b} & =\{\phi: I \rightarrow \phi \mid z(x) \text { is smooth, } \phi(x): 0 \text { for } x>b \text { and } \phi \text { satisfies }(2.1)\} \\
\gamma_{k}^{\mu}(\phi) & =\sup _{0<x<a,}\left|\left(x^{-1} D\right)^{k}\left[x^{-\mu-\frac{1}{2}} \phi(x)\right]\right|<\infty \text {, for each } k=0,1,2 \ldots
\end{aligned}
$$

$E_{L, b}$ is a linear space to which we assign the topology generated by the couritable set of seminorms $\gamma_{k}^{\mu}$. $b_{\mu, b}$ is a sequentially complete countably multi-normed space [2].

Classically, for $\mu+\frac{1}{2} \geq 0$, the finite Harkel transform of a testing function $\Phi$ in $B_{\mu, b}$ is defined as

$$
\Phi\left(\lambda_{n}\right)=\delta_{0}^{b} \phi(x) \sqrt{\lambda_{n}} \times J_{\mu}\left(\lambda_{n} x\right) d x, n=1,2,3, \ldots,
$$

where as usual $v_{\mu}$ derotes the Bessel function of the first kind of order $\mu$ and $\lambda_{n}$ $(n=1, c, 3, \ldots)$ are positive roots of $J_{\mu}(b z)=0$ (arranged in asceriding order of magnitude). However, $\Phi\left(\lambda_{r_{1}}\right)$ can be extended to the analytic function of the complex variable $z=y+i w$ by

$$
\phi(z)=0^{j} \phi(x) \sqrt{x z} J_{\mu}(x z ; d x .
$$

Note that $\Phi(z)$ is ar analytic function on the finite $z$-plane except for a brarich point at $z=0[4, p .145$ ?. Henceforth, the finite Hankel transform of a testing function $\phi$ in $B_{\mu, b}$ shall be defined as the analytic function $\Phi(z)$ given in (2.2) and denoted by $h_{\mu} \phi=\Phi$.

For a given real number $b>0, Y_{\mu, b}$ is the space of functions $\Phi(z)$ which satisfy:

$z^{-\mu-\frac{1}{2}} \Phi(z)$ is an even entire function of $z$ and for each non-negative integer $k$, the quantity 


$$
a_{b, k}^{\mu}(\Phi)=\sup _{z}\left|e^{-b|w|} z^{2 k-\left(\mu+\frac{1}{2}\right)} \Phi(z)\right|
$$

is firite. The topology of $Y_{\mu, b}$ is the one generated by using the $a_{b, k}^{\mu}$, $k=0,1,2, \ldots$, as seminorms. $Y_{\mu, b}$ is a sequentially complete cuuntably normed space. For further properties of these spaces one can look into Zemanian $[4,2]$.

For a given testing function $\Phi$ in $Y_{\mu, b}$, consider the function

$$
\phi(x)=\sigma^{j^{\infty}} \Phi(y) \sqrt{x y} J_{\mu}(x y) d y=h_{\mu}^{-1}[\Phi] .
$$

Then for $k \geq-\frac{1}{2}$, Zelliarian [2, Theoren 1] has proved:

Thecrem 2.1. For $\mu \geq-\frac{1}{2}, h_{\mu}$ is an isonorphism from $B_{\mu, b}$ onto $Y_{\mu, b}$.

Here isomorphism means topological isomorphism. Henceforth, the symbol $\Phi$ shall be used to denote a testing function in $Y_{\mu, b}$ whose pre-image is a testing functior. $\phi$ ir: $B_{1,}, b^{\text {. }}$

For a given i in $Y_{\mu, b}$, the classical inverse of the finite Hankel transform (2.2a) is a Fourier-Bessel series of the form, $[6,7]$,

$$
\frac{2}{b^{2}} \sum_{n=1}^{\infty}\left(x / \lambda_{n}\right)^{\frac{1}{2}} \frac{J_{\mu}\left(x \lambda_{n}\right)}{J_{u+1}^{2}\left(b \lambda_{n}\right)} \phi\left(\lambda_{n}\right) \text {. }
$$

Since satisfies (2.3), we have

$$
\left|\Phi\left(\lambda_{n}\right)\right| \leq \frac{A_{k \mu}}{\lambda_{n} 2 k-\left(\mu+\frac{1}{2}\right)}, k=0,1,2, \ldots, n=1,2,3, \ldots,
$$

where $A_{k \mu}$ is constant. Also $\left(x / \lambda_{n}\right)^{\frac{1}{2}}\left[J_{u}\left(x \lambda_{n}\right) / J_{\mu+1}^{2}\left(b \lambda_{n}\right)\right]$ is smocth and bounded on $0<\lambda_{n} x<\infty$, for $\mu=-\frac{1}{2}$. Consequently, the Fourier-Bessel series (2.5) converges absolutely anc uniformly in $x$ for ail $x>0$. Let us write

$$
\psi(x)=\frac{2}{b^{2}} \sum_{n=1}^{\infty}\left(x / \lambda_{n}\right)^{\frac{1}{2}} \frac{J_{\mu}\left(x \lambda_{n}\right)}{J_{\mu+1}^{2}\left(b \lambda_{n}\right)} \Phi\left(\lambda_{n}\right) \text {, }
$$

ther.

$\mid\left(x^{-1}[)^{k} x^{-\mu-\frac{1}{2}}+(x)\left|=\frac{2}{b^{2}}\right| \sum_{n=1}^{\infty}\left(x \lambda_{n}\right)^{-\mu-k} \frac{J_{\mu+k}\left(x \lambda_{n}\right)}{J_{\mu+1}^{2}\left(b \lambda_{n}\right)} \lambda_{n}^{2 k+\mu-\frac{1}{2}} \Phi\left(\lambda_{n}\right) \mid\right.$.

Since $\phi\left(\lambda_{n}\right)$ is of rapid descent as $\lambda_{n} \rightarrow \infty$ and $\left(x \lambda_{n}\right)^{-\mu-k_{j}}{ }_{\mu+k}\left(x \lambda_{n}\right)$ is smooth and bounded on $(0, \infty)$ for $\mu \geq-\frac{1}{2}$, it follows that the right-hand side of (2.6) converges absolutely and uniformly for all $x>0$ and for every $k=0,1,2 \ldots$ Hence the left-hand side is coritinuous and bounded on $0<x<\infty$ for each $k=0,1,2, \ldots$ Hence

$$
\gamma_{k}^{\mu}(\Psi), \infty, k=0,1,2, \ldots
$$

Moreover, $\left(x^{-1} D\right)^{k}\left(x^{-\mu-\frac{1}{2}} \psi(x)\right)=x^{-\mu-\frac{1}{2}}\left[a_{k 0} \frac{\psi}{x^{2 k}}+a_{k 1} \frac{D \psi}{x^{2 k-1}}+\ldots+a_{k k} \frac{D^{k} \psi}{x^{k}}\right]$,

where the $a_{k i}$ denote constants ar,d $C=\frac{d}{d x}$. So we see that the Fourier-Bessel series defines an infinitely differentiable function $\psi(x)$ satisfying $\gamma_{k}^{\mu}(\Psi)<\infty$ for each $k=0,1,2 \ldots$. But $\psi$ maay not be in $B_{\mu, b}$ as $\psi$ may not be zero for $x>b$. But

$$
\phi(x)=\lim _{\varepsilon \rightarrow 0^{+}} \lambda_{\varepsilon}(x) \Psi(x) \varepsilon B_{\mu, b},
$$


where $\lambda_{\varepsilon}(x)$ is defined as:

for $0<\varepsilon<b / 4$, and

$$
\lambda_{\varepsilon}(x)= \begin{cases}E(x / 2 \varepsilon), & 0<x<2 \varepsilon \\ 1, & 2 \varepsilon \leq x \leq b-2 \varepsilon \\ 1-E\left(\frac{x-b+2 \varepsilon}{2 \varepsilon}\right), & b-2 \varepsilon<x<b, \\ 0, & x \geq b,\end{cases}
$$

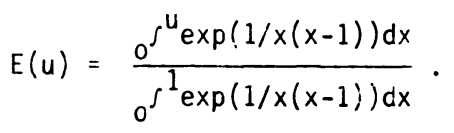

Note that $\lambda_{\varepsilon}(x)$ is a multiplier in $B_{\mu, b}$ for each $0<\varepsilon<b / 4$ since, for any $\phi \leq B_{\mu, b}$, we have

$$
\gamma_{k}^{u}\left(\lambda_{\varepsilon} \phi\right) \leq \sum_{n=0}^{k}\left(\begin{array}{l}
k \\
n
\end{array}\right) \gamma_{n}^{\mu}(\phi) \sup _{0<x<b}\left|\left(x^{-1} D\right)^{k-n} \lambda_{e}(x)\right| .
$$

Now pick $X$ such that $0<X<2 \varepsilon$. Then

$$
\sup _{x<x<b}\left|\left(x^{-1} D\right)^{n+1} \lambda_{\varepsilon}(x)\right|<\infty,
$$

and

$$
\sup _{0<x<X}\left|\left(x^{-1} D\right)^{m+1} \lambda_{\varepsilon}(x)\right| \leq A \sup _{0<x<x}\left|\left(x^{-1} D\right)^{m-1}\left[x^{-1} \exp \left(\frac{4 \varepsilon}{x(\hat{\varepsilon} \varepsilon-x}\right)\right]\right|,
$$

where $A$ is a constant.

So we see that $\gamma_{k}^{\mu}\left(\lambda_{\varepsilon} \phi\right)<\infty$. Also $\lambda_{\varepsilon}(x)$ is smooth on $(0, \infty)$. Hence $\lambda_{\varepsilon} \phi \varepsilon B_{\mu, b}$. It is easy to see that

$$
\lim _{\varepsilon \rightarrow 0^{+}} \lambda_{\varepsilon}(x) \phi(x)=\phi(x) \text {, for any } \phi \text { in } B_{\mu, b} .
$$

3. GENERALIZED FUNCTION SPACES $B_{\mu, b}^{\prime}$ AND $Y_{\mu, b}^{\prime}$.

The spaces $B_{\mu, b}^{\prime}$ and $Y_{\mu, b}^{\prime}$ are the dual spaces of $B_{\mu, b}$ and $Y_{\mu, b}$ respectively. We shall use only the weak topology of $B_{\mu, b}^{\prime}$, that is, the topology assigned to it by the seminorms

$$
\rho_{\phi}(f)=|\langle f, \phi\rangle|, \phi \varepsilon B_{\mu, b}, f \in B_{\mu, b}^{\prime} .
$$

Since $B_{\mu, b}$ is a sequentially complete countably normed space, $B_{\mu, b}^{\prime}$ is also sequentially complete [4, Theorem 1.83]. Similarly, we equip $\gamma_{\mu, b}^{\prime}$ with the weak topology generated by the seminorms $\zeta_{\Phi}(F)=|\langle F, \Phi\rangle|, \Phi \in Y_{\mu, b}, F \varepsilon Y_{\mu, b}^{\mu, b}$ $Y_{\mu, b}^{\prime}$ is a sequentially complete space.

We now construct a generalized function in $B_{\mu, b}^{\prime}$ which is not in $D^{\prime}(I)$. Let $\left\{\tau_{n}\right\}$ be a monotone increasing sequence of positive numbers with limit $b+1$. For every $\phi \in B_{\mu, b}$, the formula

$$
<f, \phi>=\sum_{n=1}^{\infty} \phi\left(\tau_{n}\right)
$$

is easily seen to define a gerieralized function $f$ in $B_{\mu, b}^{\prime}$. On the other hand, if $\phi$ is an arbitrary testirig function in $D(I)$, then $\Sigma_{n} \phi\left(\tau_{n}\right)$ is in general an 
infinite sum and it need not be convergent.

Note that

(i) $B_{\mu, b}^{\prime}$ contains every regular distribution that corresponds to a function which is Lebesgue integrable on $0<x<b$. In this case we have

$$
\left\langle f, \phi>={ }_{0} f^{b} f(x) \phi(x) d x, \phi \varepsilon B_{\mu, b}\right. \text {. }
$$

(ii) If $f$ is a tempered distribution whose support is contained in $[X, a)$ for some $x>0$, then $f \in B_{\mu, b}^{\prime}$.

(iii) Similarly, every regular distribution $F$, which can be defined by a locaily integrable function $F(y)$ through the equation

$$
<F, \Phi>=0_{0}^{\int^{\infty}} F(y) \Phi(y) d y
$$

for every $\Phi$ in $Y_{\mu, b}$, belongs to $Y_{\mu, b}^{\prime}$. Note that $F(y)$ need not be integrable over $0<y<\infty$, though typically it would be of slow growth, i.e., for some integer $N>0, y^{-N} F(y) \rightarrow 0$ as $y \rightarrow \infty$.

4. FINITE HANKEL TRANSFORMATION OF $B_{\mu, b}^{\prime}$.

Henceforth, we assume that $\mu \geq-\frac{1}{2}$. For $f \in B_{\mu, b}^{\prime}, \phi \varepsilon B_{\mu, b}$ and $\Phi={ }^{\prime}{ }_{\mu} \phi \varepsilon Y_{\mu, b}$, we define the finite Hankel transform $F=n_{\mu} f$ by

$$
\langle F, \Phi\rangle=\langle f, \phi\rangle \text {. }
$$

The above equation also defines the inverse Hankel transform $f=\dot{n}_{\mu}^{-1} F$. From Theorem 2.1 one readily obtains:

Theorem 4.1. $h_{\mu}$ is an isomorphism from $B_{\mu, b}^{\prime}$ onto $Y_{\mu, b}^{\prime}$.

Example 1. The finite Hankel transform of the delta function $\delta(x-k)$ is given by the equation (4.1):

$$
\begin{aligned}
<h_{\mu} \delta(x-k), \Phi(z)> & =\langle\delta(x-k), \phi(x)>\text {, for } 0<k<b, \\
& =<\delta(x-k),{ }_{0}^{\delta^{\infty}} \Phi \sqrt{x \bar{y}} \mathrm{~J}_{\mu}(x y) d y>\text {, (using (2.4)) } \\
& ={ }_{0} \delta^{\infty} \Phi(y) \sqrt{\mathrm{ky}} \mathrm{J}_{\mu}(k y) d y<\infty .
\end{aligned}
$$

This defines a regular distribution $F(z)=(k z)^{\frac{1}{2}} J_{\mu}(k z)$ in ${\underset{\mu}{1}, b}_{1}$. Consequently, $h_{\mu} \delta(x-k)=(k z)^{\frac{1}{2}} J_{\mu}(k z)$, for $0<k<b$.

Example 2. The finite Hankel transform of $\delta(x-k)$ for $k>b$ is the "Zero" generalized function in $Y_{\mu, b}^{\prime}$, since $\langle\delta(x-k), \phi(x)\rangle=\phi(k)=0$ for all $\phi$ in $B_{\mu, b}$. Example 3 . The finite Hankel transform of the generalized function in $B_{\mu, b}^{\prime}$ defined by (3.1) is the generalized function defined by the series

$$
F=\sum_{n=1}^{\infty} \sqrt{y \tau_{n}} J_{\mu}\left(y \tau_{n}\right)
$$

since, for $\Phi \in Y_{\mu, b}$,

$$
\begin{aligned}
\langle F, \Phi\rangle & =\sum_{n=1}^{\infty} 0^{\int^{\infty}} \Phi(y) \sqrt{\tau_{n} y} J_{\mu}\left(\tau_{n} y\right) d y \\
& =\sum_{n=1}^{\infty} \phi\left(\tau_{n}\right)=\langle f, \phi\rangle
\end{aligned}
$$

Example 4. Suppose $f$ is a regular generalized function, corresponding to a Lebesgue integrable function over $(0, b)$, in $B_{\mu, b}^{\prime}$. Than the ordinary finite Hankel transform of $f$ is given by

$$
{ }_{0} \int^{b} f(x) \sqrt{\lambda_{n} x} \mho_{\mu}\left(\lambda_{n} x\right) d x ; n=1,2,3 \ldots .
$$


Since $f$ is integrable over $(0, b)$, its finite Hankel transform may be extended to the analytic function

$$
F(z)=\int_{0}^{b} f(x) \sqrt{z x} J_{\mu}(z x) d x .
$$

We show that $F=h_{\mu}(f)$. Since $f$ is a regular generalized function,

$$
\begin{aligned}
\left\langle h_{\mu} f, \Phi\right\rangle & =\langle f, \phi\rangle \\
& =0^{\int^{b}} f(x) \phi(x) d x \\
& ={ }_{0}^{\int^{b}} f(x)\left(0_{0} \int^{\infty} \Phi(y) \sqrt{x y} J_{u}(x y) d y\right) d x \quad \text { (using (2.4)). }
\end{aligned}
$$

Since the integrand $f(x)_{\Phi}(y)(x y)^{\frac{1}{2}} J_{\mu}(x y)$ is absolutely integrable over the donia in $0<x<b, 0<y<\infty$, the order $c^{+}$integration may be changed, and we obtain

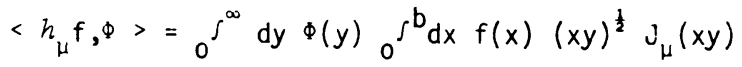

$$
\begin{aligned}
& =\langle F, \Phi\rangle \text {. }
\end{aligned}
$$

Note that $F\left(\lambda_{n}\right)={ }_{0} \delta^{b} f(x)\left(\lambda_{n} x\right)^{\frac{1}{2}} J_{\mu}\left(\lambda_{n} x\right) d x$, gives that $\left|F\left(\lambda_{n}\right)\right|$ is bounded.

Hence, for any $\dot{Q}$ in $Y_{\mu, b}$, equation (2.3) ensures that the series $\sum_{n=1}^{\mu} F\left(\lambda_{n}\right) \Phi\left(\lambda_{n}\right)$

converges. Furthermore, if a sequence $\left\{\Phi_{m}\right\}$ converges in $Y_{\mu, b}$ then the sequence of numbers $\left\{\sum_{2}^{\infty} F\left(\lambda_{n}\right) \Phi_{n !}\left(\lambda_{n}\right)\right\}$ also converges. Hence, the sum $\sum_{1}^{\infty} F\left(\lambda_{n}\right) \Phi\left(\lambda_{n}\right)$ defines a

continuous 1 inear functional on $Y_{\mu, b}^{\prime}$.

Next we investigate a representation for the finite Hankel transform of a generalized function in $B_{\mu, b}^{\prime}$. Let $D(0, b)$ be the space of infinitely differentiable furictions on $(0, b)$ with compact support contained in $(0, b)$. The topology of $O(0, b)$ is that which makes its dual $D^{\prime}(0, b)$ of Schwartz's distribution. Then $D(0, b) \subset B_{\mu, b}$ and $h_{\mu}$ maps $D(0, b)$ into a subspace of $Y_{\mu, b}$. Let $W$ be the subspace of $Y_{\mu, b}$ onto which $D(0, b)$ is mapped. Then we have

Theorem 4.2. For any generalized function $f$ in $B_{\mu, b}^{\prime}$, there exists a continuous function $F(y)$ of siow growth such that the finite Hankel transform $n_{\mu} f$ of $f$ restricted to $w_{i}$ is equivalent, in the functional sense, to the regular generalized function $F$ in $Y_{\mu, b}^{\prime}$.

Proof. For a given generalized function $f$, there exists an integer $r \geq 0$ and $a$ continuous function $h(x)[5$, Theorem 3.4.2] such that

$$
\langle f, \phi\rangle=\left\langle D^{r} h, \phi\right\rangle \text {, for every } \phi \text { in } D(0, b) \text {. }
$$

We take $h=0$ outside $(0, b)$. Then using (2.4), we have, for $\phi \in D(0, b)$,

$$
\begin{aligned}
\langle F, \Phi\rangle & =\left\langle h_{\mu} f, n_{\mu} \phi\right\rangle=\langle f, \phi\rangle=\left\langle D^{r} r_{1, \phi}\right\rangle \\
& =(-1)^{r}\left\langle h(x), D^{r} \phi(x)\right\rangle
\end{aligned}
$$




$$
\begin{aligned}
& =(-1)^{r} \int_{0}^{b} d x h(x) D^{r} \phi(x) \\
& =(-1)^{r} \int_{0}^{f^{b}} d x h(x)_{0}^{f^{\infty}} d y \Phi(y) \frac{\partial^{r}}{\partial x^{r}}\left(\sqrt{x y} J_{\mu}(x y)\right) \\
& =(-1)^{r} \int_{0}^{b} d x h(x) \delta_{0}^{f^{\infty}} d y \Phi(y) \sum_{i=0}^{r}\left[(-1)^{i} a_{i}(\mu) y^{i} x^{i-r} \sqrt{x y} J_{\mu+i}(x y)\right] \\
& =\sum_{i=0}^{r}(-1)^{i+r_{a_{j}}(\mu)} \delta_{0} f^{b} d x x^{i-r_{h}(x)\left[\int_{0} \int^{\infty} d y \Phi(y) y^{i} \sqrt{x y} J_{\mu+i}(x y)\right]}
\end{aligned}
$$

where $a_{i}(\mu)$ is a constant depending on $\mu$, for each i. If $g_{i-r}(x)=x^{i-r_{h}(x)}$, then $g_{i-r}(x)$ is continuous on $(0, \infty)$ and $g_{i-r}(x)=0$ outside $(0, b)$. Since $\Phi(y)$ is of rapid descerit and $(x y)^{\frac{1}{2}} J_{\mu+i}(x y)$ is bounded on $0<x y<\infty$, the order of integration in (4.3) may be interchanged. Therefore, (4.3) becomes

$$
\langle F, \Phi\rangle=\sum_{i=0}^{r}(-1)^{i+r_{a_{i}}(\mu)} \delta_{0}^{\delta^{\infty}} d y \Phi(y) y^{i} h_{\mu+i}\left[g_{i-r}(x)\right] .
$$

Denote $h_{\mu+i}\left[g_{i-r}(x)\right]$ by $G_{i-r}^{\mu+i}(y)$, then for $\Phi \in W$,

$$
\langle F, \Phi\rangle=\left\langle\sum_{i=0}^{r}(-1)^{i+r} a_{i}(\mu) y^{i} G_{i-r}^{i+\mu}(y), \Phi(y)\right\rangle .
$$

Clearly, the continuous function

$$
G_{i-r}^{i+\mu}(y)=o^{f^{b}} g_{j-r}(x)(x y)^{\frac{1}{2}} J_{\mu+1}(x y) d x,
$$

may be extended to an analytic function. Equation (4.4) gives

$$
h_{\mu} f \mid W=\sum_{i=0}^{r}(-1)^{i+r_{a_{i}}(\mu) y} G_{i-r}^{i+\mu}(y)=F(y) .
$$

Finally since, $\left|G_{i-r}^{i+\mu}(y)\right|<\infty$, it is obvious that $F(y)$ is of slow growth.

Example 5. From Example 1, we know that $h_{\mu} \delta(x-k)=(k z)^{\frac{1}{2}} J_{\mu}(k z)$, for $0<k<b$. On the other hand, if we define

$$
h(x)=\left\{\begin{array}{l}
0, \text { for } x \geq k, \\
x-k, \text { for } x>k,
\end{array}\right.
$$

we obtain from Theorem 4.2

It is easily seen that

$$
h_{\mu} \delta(x-k)=F(y)=a_{0}(\mu) G_{-2}^{\mu}(y)-a_{1}(\mu) y G_{-1}^{\mu+1}(y)+a_{2}(\mu) y^{2} G_{0}^{\mu+2}(y) .
$$

Also

$$
a_{0}(\mu)=\mu^{2}-\frac{1}{2}, a_{1}(\mu)=2(\mu+1), \text { and } a_{2}(\mu)=1 \text {. }
$$

$$
\begin{aligned}
& G_{-2}^{\mu}(Y)=k^{\delta^{b}}\left(\frac{1}{x}-k / x^{2}\right)(x y)^{\frac{1}{2}} J_{\mu}(x y) d x, \\
& G_{-1}^{\mu+1}(y)=k^{S^{b}}\left(1-\frac{k}{x}\right)(x y)^{\frac{1}{2}} u_{\mu+1}(x y) d x,
\end{aligned}
$$

and

$$
G_{0}^{\mu+2}(y)=k^{S^{b}(x-k)(x y)^{\frac{1}{2}} J_{\mu+2}(x y) d x .}
$$




$$
F(y)=(y k)^{\frac{1}{2}} J_{\mu}(y k)-\frac{1}{2}\left(1+\frac{k}{b}\right)(y b)^{\frac{1}{2}} J_{\mu}(y b)+\left(1-\frac{k}{b}\right) y(y b)^{\frac{1}{2}} J_{\mu}^{\prime}(y b) .
$$

This is ancther representation of $h_{\mu} \delta(x-k)$. It can be shown that this representation is equivalent to the one given in example 1 , since

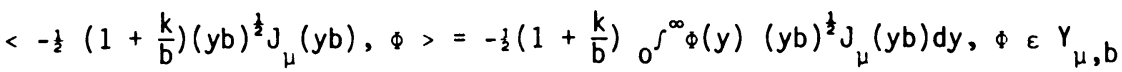

$$
\begin{aligned}
& =-\frac{1}{2}\left(1+\frac{k}{b}\right) \phi(b)=0 \text {. }
\end{aligned}
$$

Note. $\phi(b)=0$ follows from the continuity property.

Thus $\left(1-\frac{k}{b}\right)<y(y b)^{\frac{1}{2}} J_{\mu}^{\prime}(y b), \Phi>=\left(\left(1-\frac{k}{b}\right){ }_{0}^{f^{\infty}} y \Phi(y)(y b)^{\frac{1}{2}} J_{\mu}^{\prime}(y b) d y\right.$.

From (2.4) we have

Therefore,

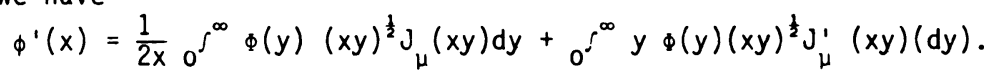

So we have

$$
\phi^{\prime}(b)=\frac{1}{2 b} 0_{0}^{S^{\infty}} \Phi(y)(\text { by })^{\frac{1}{2}} J_{\mu}(\text { by }) d y+{ }_{0}^{S^{\infty} y} \Phi(y)(\text { by })^{\frac{1}{2}} J_{\mu}^{\prime}(\text { by })(d y) .
$$

Hence

$$
\left(1-\frac{k}{b}\right)<y(b y)^{\frac{1}{2}} J^{\prime}{ }_{\mu}(b y), \Phi>=\left(1-\frac{k}{b}\right)\left[\phi^{\prime}(b)-\frac{1}{2 b} \phi(b)\right]=0 .
$$

$$
<F, \Phi>=\left\langle(y k)^{\frac{1}{2}} J_{\mu}(y k), \Phi(y)>.\right.
$$

Thus we get the same result as derived in Example 1.

Example 6. We have shown in Example 2 that $h_{j} \delta(x-k)=0$ for $k>b$. This also follows from Theorem 4.2. Take $r=2$ and define $h(x)=0$ for $x \leq k$ and $h(x)=x-k$ for $x>k$. It is easily seen that $G_{-2}^{\mu}(y)=G_{-1}^{\mu+1}(y)=G_{0}^{\mu+2}(y)=0$, hence $F(y)=0$.

Corollary 4.3. For any generalized function $f$ in $B_{\mu, b}^{\prime}$ with $r, h(x)$ and $F(y)$ defined as in the proof of Theorem 4.2, we have

$$
F(y)=\left\langle D^{r} h(x), \lambda_{\varepsilon}(x)(x y)^{\frac{1}{2}} J_{\mu}(x y)>\text {, as } \varepsilon \rightarrow 0^{+}\right. \text {. }
$$

Proof. Theorem 4.2 gives

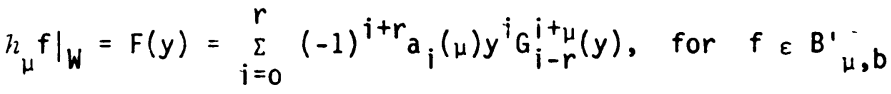

$$
\begin{aligned}
& =(-1)^{r}{ }_{0}^{b} d x \sum_{0}^{r}(-1)^{i} a_{i}(\mu) x^{-r}(x y)^{i}(x y)^{\frac{1}{2}} J_{\mu+i}(x y) \\
& =(-1)^{r}{ }_{0} s^{b} \frac{\partial^{r}}{\partial x^{r}}\left[\sqrt{x y} J_{\mu}(x y)\right] h(x) d x \\
& =(-1)^{r}{ }_{0} f^{b} \lambda_{\varepsilon}(x) \frac{\partial^{r}}{\partial x^{r}}\left[\sqrt{x y} J_{\mu}(x y)\right] h(x) d x \text { as } \varepsilon \rightarrow 0^{+} \\
& =(-1)^{r}\left\langle h(x), \lambda_{\varepsilon} \frac{\partial^{r}}{\partial x^{r}}[\sqrt{x y} \underset{\mu}{\mathcal{H}}(x y)]>\text { as } \varepsilon \rightarrow \delta\right. \text {. }
\end{aligned}
$$

But since $\lambda_{\varepsilon}(x)=1$ on $(0, b)$ as $\varepsilon \rightarrow 0^{+}$(and $h(x)=0$ outside $(0, b)$ ), the order of differentiation may be interchanged in the preceding equation to give

$$
\begin{aligned}
F(y) & =(-1)^{r}<h(x), D_{x}^{r}\left[\lambda_{\varepsilon}(x)(x y)^{\frac{1}{2}} J_{\mu}(x y)\right]>\text { as } \varepsilon \rightarrow 0^{+}, \\
& =\left\langle D_{x}^{r} h(x), \lambda_{\varepsilon}(x)(x y)^{\frac{1}{2}} J_{\mu}(x y)>, \quad \text { as } \varepsilon \rightarrow 0^{+},\right.
\end{aligned}
$$

(from the definition of distributional differentiation). 
Example 7. While calculating the finite Hankel transform of $\delta(x-k), 0<k<b$ (Example 5) using the method of Theorem 4.2, it was necessary to evaluate certain integrals to find $F(y)$. This may be avoided by using the above Corollary. From the definition of $h(x)$, we see that

and

$$
D h(x)= \begin{cases}0, & 0<x \leq k, \\ 1, & x>k,\end{cases}
$$

Hence, (4.7) gives

$$
D^{2} h(x)=\delta(x-k)
$$

$$
\begin{aligned}
F(y) & =\lim _{\varepsilon \rightarrow 0^{+}}<\delta(x-k), \lambda_{\varepsilon}(x)(x y)^{\frac{1}{2}} J_{\mu}(x y)> \\
& =\lim _{\varepsilon \rightarrow 0^{+}} \lambda_{\varepsilon}(k)(k y)^{\frac{1}{2}} J_{\mu}(k y) \\
& =(k y)^{\frac{1}{2}} J_{\mu}(k y), \text { since } \lambda_{\varepsilon}(k)=1 \text { as } \varepsilon \rightarrow 0^{+} .
\end{aligned}
$$

\section{SOME STKUCTURE THEOREMS}

In this section we shall obtain representations for members of $E_{\mu, b}, B_{\mu, b}^{\prime}$ and $Y_{\mu, b}^{\prime}$ under suitable conditions. Note that the structure formila given by Theorem 4.2 is valid only when $F \in Y_{\mu, b}^{\prime}$ is restricted to $W$, a subspace of $Y_{\mu, b}$. Here we will obtain a more general result, viz., a structure formula shall be established for $F \in Y_{\mu, b}^{\prime}$ restricted to a larger subspace than $W$ of $Y_{\mu, b}$. This section is very similar to section 3.4 of Zemanian [5, p. 86-93], consequently the corresponding results will be stated without proof or, perhaps, with only an indication of the proof. To begin with, we define certain spaces associated with $B_{\mu, b}$. Definition 5.1. We define the spaces $B_{\mu, b}^{0}, C_{\mu, b}^{0}$ and $B_{\mu, b}^{(1)}$ by

$$
\begin{aligned}
& B_{\mu, b}^{0}=\left\{\phi \in B_{\mu, b}: \phi=o\left(x^{\mu+\frac{1}{2}}\right) \text { as } x \rightarrow 0^{+}\right\}, \\
& C_{\mu, b}^{0}=\{g:(0, \infty) \rightarrow \phi \mid g \text { is continuous on }(0, \infty), g=0 \text { for } x>b \\
& \left.g(x)=o\left(x^{\mu+\frac{1}{2}}\right) \text { as } x \rightarrow 0^{+}\right\},
\end{aligned}
$$

and

$$
B_{\mu, b}^{(1)}=\left\{\phi \in B_{\mu, b}: \phi^{\prime}=0\left(x^{\mu+3 / 2}\right) \text { as } x \rightarrow 0^{+}\right\} \text {. }
$$

$B_{\mu, b}^{0}$ carries the natural topology induced on it by $B_{\mu, b}$. Note that

$$
\phi \varepsilon B_{\mu, b} \rightarrow \phi=0\left(x^{\mu+\frac{1}{2}}\right) \text {, as } x \rightarrow 0^{+} \text {. }
$$

This is true because $\gamma_{0}^{\mu}(\phi)=\sup _{0<x<b}\left|x^{-\mu-\frac{1}{2}} \phi(x)\right|<\infty$.

We prove an interesting property of functions in $B_{\mu, b}^{0}$ in

Lemma 5.2 . Let $\phi \varepsilon \mathrm{B}_{\mu, \mathrm{b}}^{0}$. Then

$$
\phi=o\left(x^{\mu+5 / 2}\right) \text { as } x+0^{+} \text {. }
$$

Proof. Let $\phi \in B_{\mu, b}^{0}$. Now

$$
\left(t^{-1} D_{t}\right)\left[t^{-\mu-\frac{1}{2}} \phi(t)\right]=t^{-\mu-\frac{1}{2}}\left[\frac{\phi^{\prime}}{t}-\left(\mu+\frac{1}{2}\right) \frac{\phi}{t^{2}}\right] \text {. }
$$


Write $n(t)=\frac{\phi^{\prime}}{t}-\left(\mu+\frac{1}{2}\right) \frac{\phi}{t^{2}}$. Clearly $n(t)$ is a smooth function on $(0, \infty)$ and $n(t)=0$, for $t>$ b. Also, $\gamma_{k}^{\mu}\left(r_{1}\right)=\gamma_{k+1}^{\mu}(\phi)<\infty$, for each $k=0,1,2, \ldots$

Hence, $n(t) \in B_{\mu, b}$. Therefore, $n(t)=o\left(t^{\mu+\frac{1}{2}}\right)$, as $t+0^{+}$.

Hence

$$
\frac{d}{d t}\left(t^{-\mu-\frac{1}{2}} \phi(t)\right)=0(t), \text { as } t \rightarrow 0^{+} .
$$

Integrating $(5.6)$, we obtain

$$
t^{-\mu-\frac{1}{2}} \phi(t)=0\left(t^{2}\right), \text { as } t+0^{+},
$$

proving Lemma 5.2.

We assign a norm to the space $c_{\mu, b}^{0}$ by

$$
\left\|j\left|\|_{0}=\sup _{x \varepsilon(0, b)}\right| x^{-\mu-\frac{1}{2}} g(x) \mid \text {, for each } g \varepsilon c_{\mu, b}^{0}\right. \text {. }
$$

Thus $c_{\mu, b}^{0}$ becomes a topological vector space. We need the following lemma which is stated without proof since the proof is identical to the proof of [5, Lemma 1, p. 88]. Lemma $5.3 . \quad B_{\mu, b}^{0}$ is a dense subset of $C_{\mu, b}^{0}$.

The following proposition gives an integral representation for the functions in $B_{\mu, b}^{0}$.

Proposition 5.4. Let $\phi \in B_{\mu, b}^{0}$. Then $\phi$ satisifies the integral equation

$$
\phi(x)=\sigma_{0}^{b} u(x, t)\left(t^{-1} D_{t}^{2}\right)\left(t^{-\mu-\frac{1}{2}} \phi(t)\right) d t,
$$

where

$$
u(x, t)=x^{\mu-\frac{1}{2}} u^{\star}(x, t),
$$

and

$$
u^{*}(x, t)= \begin{cases}\frac{x^{3}}{2 b^{2}} t\left(t^{2}-b^{2}\right), & \text { for } 0<x \leq t \leq b, \\ \frac{t^{3}}{2 b^{2}} x\left(x^{2}-b^{2}\right), & \text { for } 0<t \leq x \leq b, \\ 0, & \text { elsewhere, }\end{cases}
$$

for $0<\mathrm{x}<\infty, 0<\mathrm{t}<\infty$.

Proof. Trivial.

Next we prove that generalized functions in $B_{\mu, b}^{\prime}$ are distributional derivatives of certain continuous functions.

We start with the following boundedness property of $f \in B_{\mu, b}^{\prime}$.

For each $f \in B_{\mu, b}^{\prime}$, there exists a non-negative integer $r$ and a positive constant A such that for all $\phi \in B_{\mu, b}$,

$$
|\langle f, \phi\rangle| \leq A \max _{0 \leq k \leq r} \gamma_{k}^{\mu}(\phi)=\rho_{r}^{\mu}(\phi) \text { (say). }
$$

Suppose $f \in B_{\mu, b}^{\prime}$ is such that (5.12) is satisfied with $r=0$. Then

$$
|<f, \phi>| \leq A \sup _{0<x<b}\left|x^{-\mu-\frac{1}{2}} \phi(x)\right| \text {. }
$$

We now extend $f$, satisfying the inequality (5.13), continuously and uniquely onto the space $c_{\mu, b}^{0}$. Let $g$ in $c_{\mu, b}^{0}$ be arbitrary. Then by Lemma 5.3 , there exists a sequence $\left\{\phi_{n}\right\}$ of testing functions in $B_{\mu, b}^{0}$ such that $\phi_{n}$ converges to $g$ in 
$c_{\mu, b}^{0}$. We define $\langle f, g\rangle$ by

$$
\langle f, g\rangle=\lim _{n \rightarrow \infty}\left\langle f, \phi_{n}\right\rangle \text {. }
$$

This defines a continuous linear functional on $C_{\mu, b}^{0}$ satisfying the inequality (5.13).

Clearly $u(x, t) \in C_{\mu, b}^{0}$, hence the following definition makes sense for $f \in B_{\mu, b}^{\prime}$ satisfying $(5.13)$.

Definition 5.5. For $f \in B_{\mu, b}^{\prime}$ satisfying the inequality (5.13), define $h(t)=\langle f(x), u(x, t)\rangle$.

Note: (i) $h(t)=0$ for $t \geq b$, as $u(x, t)=0$ for $t \geq b$

(ii) $|h(t)-h(\tau)| \leq 3 A b^{2}|t-\tau|, 0<t \leq b, 0<\tau \leq b$.

Lemma 5.6. For $\phi \varepsilon B_{\mu, b},\left(\frac{1}{t} D_{t}\right)^{2}\left[t^{-\mu-\frac{1}{2}} \phi(t)\right]$ is uniformly continuous on $(0, b]$. Proof. Let

$$
n(t)=\left(\frac{1}{t} \frac{d}{d t}\right)^{2}\left[t^{-\mu-\frac{1}{2}} \phi(t)\right], \phi \varepsilon B_{\mu, b} .
$$

Then, $n(t)=0(1)$ as $t \rightarrow 0^{+}$, and $\left|n^{\prime}(t)\right|<\infty$, proving the Lemma 5.6.

Theorem 5.7. For $f \in B_{\mu, b}^{\prime}$ satisfying the condition

$$
|<f, \phi\rangle\left|\leq A \sup _{0<x<b}\right| x^{-\mu-\frac{1}{2}} \phi(x) \mid, \forall \phi \varepsilon B_{\mu, b}^{0},
$$

we have

$$
\langle f, \phi\rangle={ }_{0} \int^{b} h(t)\left(t^{-1} D_{t}\right)^{2}\left[t^{-\mu-\frac{1}{2}} \phi(t)\right] d t
$$

for every $\phi \in E_{\mu, b}^{0}$. Here, $h(t)$ is the continuous function defined by equation

(5.15). If $D_{t}\left[t^{-1} D_{t}\left(t^{-1} h(t)\right)\right]$ is Lebesgue integrable over $(0, b),(5.17)$ can be written as

for every $\phi \varepsilon B_{\mu, b}^{0}$.

$$
\langle f, g\rangle=\left\langle t^{-\mu-\frac{1}{2}} D_{t}\left(t^{-1} D_{t}\left(t^{-1} h(t)\right)\right), \phi(t)\right\rangle,
$$

Procf. The proof of (5.17) is very similar to the proof of [5, equation (9), p. 90-91] and (5.18) follows easily from (5.17).

We now generalize Theorem 5.7 for the case when $\left.|\langle f, \phi\rangle| \leq \rho_{r}^{\mu}(\phi), r\right\rangle 0$. For this we need the following:

Definition 5.8. For each non-negative integer $n$, we define the spaces $B_{\mu, b}(n), H_{\mu, b}^{(n)}$, $B_{\mu, b}^{\infty}$, and $H_{\mu, b}^{\infty}$ by

$$
\begin{aligned}
& B_{\mu, b}^{(n)}=\left\{\phi \in B_{\mu, b}: \phi^{(n)}(x)=0\left(x^{\mu+3 / 2}\right) \text { as } x \rightarrow 0+\right\}, \\
& H_{\mu, b}^{(n)}=\left\{n(x)=\frac{\phi^{\prime}(x)}{\left(\mu+\frac{1}{2}\right) x}-\frac{\phi(x)}{x^{2}}: \phi \varepsilon B_{\mu, b}^{(n)}\right\},
\end{aligned}
$$

where $\phi^{(n)}(x)=D_{\phi}^{n_{\phi}(x)}$ for $n \neq 0$ and $\phi^{(0)}(x)=\phi(x)$,

$$
B_{\mu, b}^{\infty}=\left\{\phi \in B_{\mu, b}: \phi^{(k)}(x)=0\left(x^{\mu+\frac{1}{2}}\right), \text { as } x \rightarrow 0+, \text { for each } k=0,1,2, \ldots\right\},(5.21)
$$

and

$$
H_{\mu, b}^{\infty}=\left\{n(x)=\frac{\phi^{\prime}(x)}{\left(\mu+\frac{1}{2}\right) x}-\frac{\Phi(x)}{x^{2}}: \phi \varepsilon B_{\mu, b}^{\infty}\right\} .
$$


Note that $H_{\mu, b}^{(1)} \subset B_{\mu, b}^{0}$ and $B_{\mu, b}^{0}=B_{\mu, b}^{(0)}$. Let $\phi_{0} \varepsilon B_{\mu, b}^{0}$ be such that

$$
0_{0}^{b} t^{-\mu+\frac{1}{2}} \phi_{0}(t) d t=1 \text {. }
$$

In the subsequent development we shall need the following lemma, whose proof is omitted since it is similar to that of [5, Lemma 1, p.68].

Lemma 5.9. Let $\phi_{0}$ be a fixed testing function in $B_{\mu, b}^{0}$ satisfying (5.23). Then any testing function $\phi$ in $B_{\mu, b}^{0}$ may be decomposed uniquely according to

$\phi=K_{\phi_{0}}+n$
where $n$ is in $H_{\mu, b}^{(1)}$ and the constant $K$ is given by

$$
K=0^{S} t^{-\mu+\frac{1}{2}} \phi(t) d t
$$

Suppose $f$ is a regular generalized function in $B_{\mu, b}^{\prime}$ generated by a differentiable function $f$ such that $f$ is Lebesgue integrable over $(0, b)$ and $f^{\prime}$ is bounded on $(0, b]$. Then for $n \in H_{\mu, b}^{(1)}$ we have

$$
\begin{aligned}
\langle f, n\rangle & =\int_{0}^{b} f^{\prime}(x) n(x) d x \\
& \left.=\frac{1}{\mu+\frac{1}{2}} 0^{\int b} x^{\mu-\frac{1}{2}} f(x) \frac{d}{d x}\left[x^{-\mu-\frac{1}{2}} \phi(x)\right] d x \text { (for some } \phi \in B_{\mu, b}^{(1)}\right) \\
& =-\frac{1}{\mu+\frac{1}{2}} 0^{j} b \frac{\left(x^{\mu-\frac{1}{2}} f(x)\right)^{\prime}}{x^{\mu+\frac{1}{2}}} \phi(x) d x,
\end{aligned}
$$

since $\phi(b)=0$ and $\frac{\phi(x)}{x}=0\left(x^{\mu+3 / 2}\right)$ as $x \rightarrow 0+$.

Therefore

$$
\langle f, n\rangle=\frac{1}{\mu+\frac{1}{2}}\left\langle x^{-\mu-\frac{1}{2}} D x^{\mu-\frac{1}{2}} f(x),-\phi(x)\right\rangle .
$$

But

$$
n=\frac{x^{\mu-\frac{1}{2}}}{\mu+\frac{1}{2}} D x^{-\mu-\frac{1}{2}} \phi(x)
$$

therefore,

$$
\left\langle f(x), x^{\mu-\frac{1}{2}} D x^{-\mu-\frac{1}{2}} \phi(x)>=\left\langle x^{-\mu-\frac{1}{2}} D x^{\mu-\frac{1}{2}} f(x),-\phi(x)>.\right.\right.
$$

Let

and

Then for any $\phi \in B_{\mu, b}^{(1)}$,

$$
L_{\mu}=x^{\mu-\frac{1}{2}} D x^{-\mu-\frac{1}{2}} \text {, }
$$

and

$$
L_{\mu} \phi=x^{\mu+\frac{1}{2}}\left(x^{-1} D\right)\left(x^{-\mu-\frac{1}{2}} \phi(x)\right) \varepsilon B_{\mu, b}^{0},
$$

We write the above as

$$
\gamma_{k}^{\mu}\left(L_{\mu} \phi\right)=\gamma_{k+1}^{\mu}(\phi) \text {. }
$$

Lemma 5.10. The operation $\phi+L_{\mu} \Phi$ is a continuous linear mapping of $B_{\mu, b}^{(1)}$ into $\mathrm{B}_{\mu, \mathrm{b}}^{\mathrm{O}}$. For an arbitrary $f$ in $B_{\mu, b}^{\prime}$ and any $\Phi$ in $B_{\mu, b}^{(1)}$, set 


$$
\left\langle T_{\mu} f, \phi\right\rangle=\left\langle x^{-\mu-\frac{1}{2}} D x^{\mu-\frac{1}{2}} f, \phi\right\rangle=\left\langle f,-L_{\mu} \phi\right\rangle .
$$

From (5.30) we see that $T_{\mu} f$ is a linear functional on $B_{\mu, b}^{(1)}$. Write

$$
x^{-\mu-\frac{1}{2}} D x^{\mu-\frac{1}{2}} \quad f=g
$$

We then have formally,

$$
D x^{\mu-\frac{1}{2}} f=x^{\mu+\frac{1}{2}} g .
$$

Now define,

$$
x^{-\mu+\frac{1}{2}}\left[x^{\mu+\frac{1}{2}} g\right]^{(-1)}=f .
$$

With this notation (5.26) suggests that

$$
<x^{-\mu+\frac{1}{2}}\left[x^{\mu+\frac{1}{2}} f\right]^{(-1)}, n>=\frac{1}{\mu+\frac{1}{2}}\langle f,-\phi>.
$$

Equation (5.32) defines a linear functional on $H_{H, b}^{(1)}$. This can be exterided to all of $B_{\mu, b}^{O}$ by using Lemma 5.9. Assign

$$
<x^{-\mu+\frac{1}{2}}\left[x^{\mu+\frac{1}{2}} f\right]^{(-1)}, \phi_{0}>=k_{0} \text { (arbitrary). }
$$

Then for any $\phi \in B_{\mu, b}^{0}$, we have from Lemma 5.9,

$$
\phi=K_{0}+n \text {. }
$$

Hence the operator $x^{-\mu+\frac{1}{2}}\left[x^{\mu+\frac{1}{2}} f\right]^{(-1)}$ is defined for all of $B_{\mu, b}^{0}$.

Proposition 5.11. Suppose $f \in B_{\mu, b}^{\prime}$ satisfies

$$
|<f, \phi>| \leq A \sup _{0 \leq k \leq r} \gamma_{k}^{\mu}(\Phi), \quad r \geq 1
$$

for all $\phi$ in $B_{\mu, b^{\circ}}^{0}$ Then

$$
\left.1<x^{-\mu+\frac{1}{2}}\left[x^{\mu+\frac{1}{2}}\right] f(x)\right]^{(-1)}, \phi(x)>1 \leq A \sup _{0 \leq k \leq r-1} \gamma_{k}^{\mu}(\phi),
$$

for all $\phi \in B_{\mu, b}^{0}$.

Proot. Using (5.32), the proof follows trivially for members of $H_{\mu, b}^{(1)}$. Now use Lemma 5.9 to complete the proof.

Proposition 5.12. For $f \in B_{\mu, b}^{\prime}$ satisfying

$$
|<f, \phi>| \leq A \sup _{0 \leq k \leq 1} \gamma_{k}^{\mu}(\phi), \quad \forall \phi \varepsilon B_{\mu, b}^{0},
$$

we have

$$
<f, \phi>--_{0} f^{b} h(t)\left(t^{-1} D_{t}\right)^{3}\left(t^{-\mu-\frac{1}{2}} \phi(t)\right) d t,
$$

for each $\phi$ in $B_{\mu, b}^{(1)}$ and where $h$ is a continuous function.

Proof. We know that the theorem is true for $r=0$. For $\phi \in B_{\mu, b}^{(1)}$,

$$
\langle f, \phi\rangle=-\left(\mu+\frac{1}{2}\right)<x^{-\mu+\frac{1}{2}}\left[x^{\mu+\frac{1}{2}} f\right]^{(-1)}, n>, \eta \in H_{\mu, b}^{(1)} \text { (equation (5.32)) }
$$




$$
=-\left(\mu+\frac{1}{2}\right) \delta_{0}^{b} h(t)\left(t^{-1} D_{t}\right)^{2}\left(t^{-\mu-\frac{1}{2}} n(t)\right) d t \text {, }
$$

by Theorem (5.7), since

and

$$
1<x^{-\mu+\frac{1}{2}}\left[x^{\mu+\frac{1}{2}} f\right]^{(-1)}, n>\mid \leq A \gamma_{0}^{\mu}(n) \text {, (see Proposition (5.11)) }
$$

Therefore,

$$
n(x)=o\left(x^{\mu+\frac{1}{2}}\right) \text {, as } x+0+\text {. }
$$

$$
\begin{aligned}
<f, \phi> & =-\left(\mu+\frac{1}{2}\right) o^{\cdot} b(t)\left(t^{-1} D_{t}\right)^{2}\left[t^{-\mu-\frac{1}{2}} \cdot \frac{t^{\mu+\frac{1}{2}}}{\mu+\frac{1}{2}} \frac{1}{t} \frac{d}{d t}\left(t^{-\mu-\frac{1}{2}} \phi(t)\right)\right] d t \\
& =-\sigma_{0}^{b} h(t)\left(t^{-1} D_{t}\right)^{3}\left(t^{-\mu-\frac{1}{2}} \phi(t)\right) d t .
\end{aligned}
$$

If we replace both $B_{\mu, b}^{0}$ and $B_{\mu, b}^{(1)}$ by $B_{\mu, b}^{\infty}$ and $H_{\mu, b}^{(1)}$ by $H_{\mu, b}^{\infty}$, Lemmas 5.9 , 5.10 and Propositions 5.11, 5.12 are still true. Hence by induction on $r$, we get Theorem 5.13. For $f \in B_{\mu, b}^{\prime}$ satisfying

$$
|<f, \phi>| \leq A \sup _{0 \leq k \leq r} \gamma_{k}^{\mu}(\phi), \quad\left(\forall \phi \in B_{\mu, b}^{0}\right)
$$

for some non-negative integer $r$, we have

$$
<f, \phi>=(-1)^{r} \delta_{0}^{b} h(t)\left(t^{-1} C_{t}\right)^{r+2}\left[t^{-\mu-\frac{1}{2}} \phi(t)\right] d t,
$$

for each $\phi \in B_{\mu, b}^{0}$ where $h$ is a continuous function.

The above structure theorem helps us to say more about the finite Hankel transform of elements in $B_{\mu, b}^{\prime}$.

Let $\phi \in B_{\mu, b}^{\infty}$ and $F, \Phi$ be the finite Hankel transforms of $f \in B_{\mu, b}^{\prime}$ and $\phi$ respectively. Then

$$
\begin{aligned}
\langle F, \Phi\rangle & =\langle f, \Phi\rangle \\
& =(-1)^{r-2}{ }_{0}^{b} h(x)_{0} f^{\infty}(y)^{\frac{1}{2}} \phi(y)\left(\frac{1}{x} \frac{d}{d x}\right)^{r}\left(x^{-\mu} J_{\mu}(x y)\right) d y d x
\end{aligned}
$$

(using equations (2.4) and (5.37).

Since

then

$$
(-1)^{r}\left(\frac{1}{x} \frac{d}{d x}\right)^{r}\left(x^{-\mu} J_{\mu}(x y)\right)=y^{r} x^{-\mu-r} J_{\mu+r}(x y),
$$

$$
\begin{aligned}
& \langle F, \Phi\rangle={ }_{0}^{f^{b}} h(x) \int_{0}^{S^{\infty}}(y)^{\frac{1}{2}} \phi(y) y^{r} x^{-\mu-r} J_{\mu+r}(x y) d y d x \\
& =\int_{0}^{\delta^{\infty}} y^{r} \Phi(y)_{0}^{f^{b}} \frac{h(x)}{\mu+\frac{1}{2}+r} \sqrt{x y} J_{\mu}{ }_{\mu+r}(x y) d x d y .
\end{aligned}
$$

Now let $g_{r}(x)=\frac{h(x)}{x^{\mu+\frac{1}{2}+r}}$.

Since

$$
\frac{\sqrt{x y} J_{\mu+r}(x y)}{x^{\mu+\frac{1}{2}+r}} \sim \frac{(x y)^{\mu+r+\frac{1}{2}}}{x^{\mu+\frac{1}{2}+r}} \text { as } x \rightarrow 0^{+} \text {, }
$$


so that

is well defined.

$$
G_{r}^{\mu+r}(y)=0^{\circ}{ }^{b} g_{r}(x)(x y)^{\frac{1}{2}} J_{\mu+r}(x y) d x
$$

Thus (5.40) becomes

Consequently,

$$
\begin{aligned}
<F, \Phi> & =\int_{0}^{\delta^{\infty}} y^{r} \Phi(y) G_{r}^{\mu+r}(y) d y \\
& =\left\langle y^{r} G_{r}^{\mu+r}(y), \Phi(y)\right\rangle
\end{aligned}
$$

$$
F=y^{r} G_{r}^{\mu+r}(y) \text { (in the functional sense) }
$$

is of slow growth since $\left|G_{r}^{\mu+r}(y)\right|<\infty$. We 1 ist the above as

Thecrem 5.14. For any $F \in Y_{\mu, b}^{\prime}$, there exists a continuous function $G(y)$ $\left(=y^{r} G_{r}^{\mu+r}(y)\right)$ of slow growth such that $\left.F\right|_{Y_{\mu, b}^{\infty}}$ is equivalent to $G(y)$, i.e.,

for $\Phi \in Y_{\mu, b}^{\infty}$, where $Y_{\mu, b}^{\infty}=\begin{aligned} & \langle F, \Phi\rangle= \\ & h_{\mu}\left[B_{\mu, b}^{\infty}\right] \text {. }\end{aligned}$

Furthermore, $G(y)$ may be extended to an analytic function.

\section{FURTHER PROPERTIES OF THE FINITE HANKEL TRANSFORM}

Notation: Let us write $\hat{f}=\left.f\right|_{B_{\mu, b}^{\infty}}$, for any $f \varepsilon B_{\mu, b}^{\prime}$, and likewise $\hat{F}=\left.F\right|_{Y_{\mu, b}^{\infty}}$ for any $F \in Y_{\mu, b}^{\prime}$. Also write $\hat{\phi}$ to denote the members of $B_{\mu, b}^{\infty}$ and $\hat{\phi}$ for the members of $Y_{\mu, b}^{\infty}$

For any $\hat{f}$, we have for some integer $r \geq 2$,

$$
<\hat{f, \hat{\phi}}>=(-1)^{r} \delta_{0}^{b} h(x)\left(\frac{1}{x} \frac{d}{d x}\right)^{r}\left(x^{-\mu-\frac{1}{2}} \phi(x)\right) d x,
$$

where $h(x)$ is a continuous function.

Definition 6.1 . We define a new space $H_{\mu}^{\infty}(I)$ by

$$
H_{\mu}^{\infty}(I)=\left\{\phi \in H_{\mu}: \phi^{(k)}(x)=0\left(x^{\mu+3 / 2}\right) \text { as } x \rightarrow 0^{+} \text {, for } k=0,1,2, \ldots\right\} \text {, }
$$

where $I=(0, \infty)$.

Now define $h_{b}(x)$ to be the periodic extension of period $b$ of $h(x)$ on $(0, b]$. Then for any $x \in R^{+}, h_{b}(x)=h(x-n b)$ for some positive integer $n$ such that $0<x-n b \leq b$. Associated with $h_{b}(x)$ is the regular distribution in $H_{\mu}^{\infty}{ }^{\prime}(I)$ having the value

$$
<h_{b}(x), \phi(x)>=\sum_{n=0}^{\infty} 0^{b} h(x) \phi(x+n b) d x,
$$

for any $\phi \in H_{\mu}^{\infty}(I)$.

The right-hand side of (6.2) converges, since $\phi$ is of rapid descent as $x \rightarrow \infty$. Now define a functional $f_{b}$ on $H_{\mu}^{\infty}(I)$ by 


$$
\begin{aligned}
\left\langle f_{b}, \phi\right\rangle & =(-1)^{r} \int_{0}^{\delta^{\infty}} h_{b}(x)\left(\frac{1}{x} D_{x}\right)^{r}\left(x^{-\mu-\frac{1}{2}} \phi(x)\right) d x \\
& =\sum_{n=0}^{\infty}(-1)^{r}\left[\int_{0}^{\infty} h(x-n b)\left(\frac{1}{x} D_{x}\right)^{r} \cdot\left(x^{-\mu-\frac{1}{2}} \phi(x)\right) d x\right] \\
& =\sum_{n=0}^{\infty}(-1)^{r}{ }_{n b} \delta^{(n+1) b} h(x-n b)\left(\frac{1}{x} D_{x}\right)^{r}\left(x^{-\mu-\frac{1}{2}} \phi(x)\right) d x .
\end{aligned}
$$

This defines a linear continuous functional on $H_{\mu}^{\infty}(I)$. Also for $\hat{\phi}$ in $B_{\mu, b}^{\infty}$, we have

$$
\begin{aligned}
<f_{b}, \hat{\phi}> & \left.=(-1)^{r} o^{b} h(x)\left(\frac{1}{x} D_{x}\right)^{r}\left(x^{-\mu-\frac{1}{2}} \phi(x)\right) d x \text {, (s ince } \hat{\phi}=0 \text { for } x>b\right) \\
& =\langle\hat{f}, \hat{\phi}>.
\end{aligned}
$$

So we see that $f_{b}$ is a periodic extension of $f$.

Theorem 6.2. Every $\hat{f}$ in $B_{\mu, b}^{\prime}$ may be extended to a periodic linear functional, with period $b$, on $H_{\mu}^{\infty}(I)$ which is continuous in the topology of $H_{\mu}$.

Theorem 6.3. For every $\varepsilon$ in $(0, b / 4)$, and each $f$ in $B_{\mu, b}^{\prime}$, the function

$$
F_{\varepsilon}(y)=\left\langle f(x), \lambda_{\varepsilon}(x)(x y)^{\frac{1}{2}} J_{\mu}(x y)\right\rangle,
$$

where $\lambda_{\varepsilon}(x)$ is defined by (2.7), is a smooth function of slow growth and defines a regular generalized function in $Y_{\mu, b}^{\prime}$.

Proof. The proof of the above theorem is similar to the proof of [1, Lemma 12] given by Zemanian.

Theorem 6.4. The finite Hankel transform, $h_{\mu} f$, of a generalized function $f$ in $B_{\mu, b}^{\prime}$ is the limit, as $\varepsilon \rightarrow 0$, of the family $F_{\varepsilon}(z)$ of regular generalized functions defined in Theorem 6.3.

Proof. Since $F_{\varepsilon}(z)$ is a regular functional in $Y_{\mu, b}^{\prime}$, it is sufficient to show that

$$
<h_{\mu} f, \Phi>=\left\langle F_{\varepsilon}, \Phi>\right.
$$

for each $\Phi$ in $Y_{\mu, b}$, as $\varepsilon \rightarrow 0$. For each $\Phi$ in $Y_{\mu, b}$ there exists à unique $\phi$ in $B_{\mu, b}$ given by Equation (2.4). As $\varepsilon \rightarrow 0+, \lambda_{\varepsilon}(x)=1$ on $(0, b)$.

Now we have

$$
\begin{aligned}
\left\langle F_{\varepsilon}, \Phi\right\rangle & =\left\langle f(x), \lambda_{\varepsilon}(x)(x y)^{\frac{1}{2}} J_{\mu}(x y)>, \Phi(y)>\right. \\
& ={ }_{0}^{S^{\infty}}<f(x), \lambda_{\varepsilon}(x)(x y)^{\frac{1}{2}} J_{\mu}(x y)>\Phi(y) d y \\
& =\left\langle f(x), \lambda_{\varepsilon}(x){ }_{0}^{S^{\infty}} \Phi(y)(x y)^{\frac{1}{2}} J_{\mu}(x y) d y>\right.\text { (by [8, Corollary 5.3.2b, p. 121]) } \\
& =\left\langle f(x), \lambda_{\varepsilon}(x) \phi(x)>\right. \\
& \rightarrow\langle f(x), \phi(x)>, \text { as } \varepsilon \rightarrow 0+.
\end{aligned}
$$


Consequently, $\left\langle F_{\varepsilon}, \Phi\right\rangle \rightarrow\langle f(x), \phi(x)\rangle=\left\langle h_{\mu} f, \Phi\right\rangle$, as $\varepsilon \rightarrow 0+$, for each $\Phi \varepsilon Y_{\mu, b}$

$$
\text { Since } \lambda_{\varepsilon}(x)(x y)^{\frac{1}{2}} J_{\mu}(x y)+x(0, b)(x)(x y)^{\frac{1}{2}} J_{\mu}(x z) \text {, as } \varepsilon \rightarrow 0+\text {, (where } x(0, b) \text { is }
$$

the characteristic furction of the interval $(0, b))$ and the latter is not a testing function in $B_{\mu, b}$, it is not true, in general, that the limit of $F_{\varepsilon}(z)$, as $\varepsilon \rightarrow 0$, exists as an ordinary function. Where the limit does exist as an ordinary function, it will be denoted by $F_{0}(z)$.

Corollary 6.5. If $f$ is a regular generalized function in $B_{\mu, b}^{\prime}$, then the limiting function $F_{0}(z)$ exists and is equivalent to the finite Hankel transform of $f$.

Proof. If $f$ is regular, then for each $\varepsilon$ in $(0, b / 4)$,

$$
F_{\varepsilon}(z)=\sigma_{0} b^{b} f(x) \lambda_{\varepsilon}(x)(x y)^{\frac{1}{2}} J_{\mu}(x z) d x .
$$

As $E \rightarrow 0^{+}$, we obtain

$$
F_{0}(z)=c^{s^{b}} f(x)(x y)^{\frac{1}{2}} J_{\mu}(x z) d x,
$$

and from Example 4 , we see that $F_{0}(z)$ is equivalent to $h_{\mu} f$.

Using the function $x_{(0, b)}(x),(6.5)$ can be written as

$$
F_{0}(z)=\left\langle x(0, b)(x) f(x), \lambda(x)(x z)^{\frac{1}{2}} J_{\mu}(x z)\right\rangle,
$$

where $\lambda(x)$ is a testing function in $D(R)$ such that $\lambda(x)=1$ on $(0, b]$. In this case, to calculate the finite Hankel transform we merely truncate the regular distribution in $B_{\mu, b}^{\prime}$ at $x=b$, as would be expected. In a similar way one might interpret the limit as $\varepsilon \rightarrow 0+$ of the family of the functions $F_{\varepsilon}(z)$ as a process of truncation for distributions in general, for one is replacing $f(x)$ by the distributional limit $\lambda_{\varepsilon}(x) f(x)$ as $\varepsilon \rightarrow 0+$.

Corollary 6.6. If the generalized function $f \varepsilon B_{\mu, b}^{\prime}$ has support in $(0, b]$, then the function $F_{0}(z)$ exists and is equivalent to the finite Hankel transform of $f$.

Proof. Let $\lambda(x)$ be a testing function in $D(I)$ such that $\lambda(x)=1$ on a neighborhood of the support of $f$. Then

such that,

$$
F_{\varepsilon}(z)=\left\langle f(x), \lambda_{\varepsilon}(x) \lambda(x)(x z)^{\frac{1}{2}} J_{\mu}(x z)\right\rangle
$$

$$
\lim _{\varepsilon \rightarrow 0^{+}} F_{\varepsilon}(z)=F_{0}(z)=\left\langle f(x), \lambda(x)(x z)^{\frac{1}{2}} J_{\mu}(x z)\right\rangle,
$$

since $\lambda_{\varepsilon}(x)=1$ on the support of $f$ as $\varepsilon \rightarrow 0+$. But Zemanian [1, Theorem 2] has proved that

$$
h_{\mu} f=\left\langle f(x), \lambda(x)(x z)^{\frac{1}{2}} J_{\mu}(x z)>,\right.
$$

for every functional in $H_{\mu}^{\prime}$ having compact support.

Example 8. For the distribution $\delta(x-k)$, from (6.4) we obtain

$$
\begin{aligned}
F_{\varepsilon}(z) & =\left\langle\delta(x-k), \lambda_{\varepsilon}(x)(x z)^{\frac{1}{2}} J_{\mu}(x z)\right\rangle \\
& =\lambda_{\varepsilon}(k)(k z)^{\frac{1}{2}} J_{\mu}(k z) .
\end{aligned}
$$


But for $0<k<b, \lambda_{\varepsilon}(k)=1$ as $\varepsilon \rightarrow 0+$. And for $k \geq b, \lambda_{\varepsilon}(k)=0$.

Therefore,

$$
\begin{aligned}
F_{0}(z) & = \begin{cases}\sqrt{k z} J_{\mu}(k z), & \text { for } 0<k<b \\
0, & \text { for } k \geq b,\end{cases} \\
& =h_{\mu}[\delta(x-k)] .
\end{aligned}
$$

\section{THE FOURIER-BESSEL SERIES}

Classically the inverse finite liankel transform is considered to be the Fourier-Bessel series

$$
\frac{2}{b^{2}} \sum_{n=1}^{\infty}\left(x / \lambda_{n}\right)^{\frac{1}{2}} \frac{J_{\mu}\left(x \lambda_{n}\right)}{J_{\mu+1}^{2}\left(b \lambda_{n}\right)} \cdot \Phi\left(\lambda_{n}\right)
$$

where

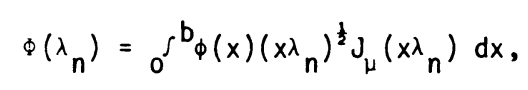

is the finite Hankel transform [7] of some function $\phi$. have

In section 2, we showed that for any $\Phi \in B_{\mu, b}$ and $\Phi\left(\lambda_{n}\right)$ given by (7.2), we

$$
\phi(x)=\frac{2}{b^{2}} \sum_{n=1}^{\infty} \lambda_{\varepsilon}(x)\left(\frac{x}{\lambda_{n}}\right)^{\frac{1}{2}} \cdot \frac{J_{\mu}\left(x \lambda_{n}\right)}{j_{\mu+1}^{2}\left(b \lambda_{n}\right)} \Phi\left(\lambda_{n}\right) \text {, as } \varepsilon \rightarrow 0 \text {. }
$$

We obtain an inversion theorem similar to (7.1) for generalized finite Harkel transforms of members in $B_{\mu, b}^{\prime}$.

Theorem 7.1 (inversion). Let $f$ be an arbitrary generalized function in $B_{\mu, b}^{\prime}$, where $\mu \geq-\frac{1}{2}$. Let $F=h_{\mu}(f)$, be the finite Hankel transform. Then in the sense of convergence in $B_{\mu, b}^{\prime}$, we have

$$
f(x)=\lim _{N \rightarrow \infty} \frac{2}{b^{2}} \sum_{n=1}^{\infty}\left(\frac{x}{\lambda_{n}}\right)^{\frac{1}{2}}\left[J_{\mu}\left(x \lambda_{r_{1}}\right) / J_{\mu+1}^{2}\left(b \lambda_{n}\right)\right] F\left(\lambda_{n}\right) .
$$

Proof. Let $\lambda(x)$ be an arbitrary testing function in $B_{\mu, b}$. We wish to prove that

$$
\left.<\frac{2}{b^{2}} \sum_{n=1}^{N}\left(\frac{x}{\lambda_{n}}\right)^{\frac{1}{2}} \frac{J_{\mu}\left(x \lambda_{n}\right)}{J_{\mu+1}^{2}\left(b \lambda_{n}\right)} F\left(\lambda_{n}\right), \phi(x)\right\rangle \rightarrow\langle f(x), \phi(x)\rangle \text {, dS } N \rightarrow \infty \text {. }
$$

Since $(x)^{\frac{1}{2}} J_{\mu}\left(x \lambda_{n}\right)$ is locally integrable over $(0, b)$,

$$
\begin{aligned}
<\frac{2}{b^{2}} \sum_{n=1}^{N}\left(\frac{x}{\lambda_{n}}\right)^{\frac{1}{2}} \frac{J_{\mu}\left(x \lambda_{n}\right)}{J_{\mu+1}^{2}\left(b \lambda_{n}\right)} F\left(\lambda_{n}\right), \phi(x)> \\
={ }_{0}^{\delta} b \frac{2}{b^{2}} \sum_{n=1}^{N} \frac{F\left(\lambda_{n}\right)}{J_{\mu+1}^{2}\left(b \lambda_{r_{1}}\right)}\left(\frac{x}{\lambda_{n}}\right)^{\frac{1}{2}} \cdot J_{\mu}\left(x \lambda_{n}\right) \phi(x) d x \\
=\sum_{n=1}^{N} \frac{2}{b^{2}} \frac{F\left(\lambda_{n}\right)}{\lambda_{n} J_{\mu+1}^{2}\left(b \lambda_{n}\right)} \Phi\left(\lambda_{n}\right), \quad \text { (from (2.2)) }
\end{aligned}
$$




$$
\begin{aligned}
& =\sum_{n=1}^{N} \frac{2}{b^{2}} \frac{\Phi\left(\lambda_{n}\right)}{\lambda_{n} j_{\mu+1}^{2}\left(b \lambda_{n}\right)} \lim _{\varepsilon \rightarrow 0+}\left\langle f(x), \lambda_{\varepsilon}(x)\left(x \lambda_{s}\right)^{\frac{1}{2}} J_{\mu}\left(x \lambda_{n}\right)>\right. \\
& \quad(\text { from Theorem 6.4) } \\
& \rightarrow<(x), \phi(x)>\text {, as } N \rightarrow \infty .
\end{aligned}
$$

We verify this inversion Theorem by means of a numerical example.

Example 9. For $0<k<b, \delta(x-k) \in E^{\prime}(I) \subset H_{\mu}^{\prime} \subset B_{\mu, b}^{\prime}$. The finite Hankel transform of $\delta(k-x)$ is,

Hence,

$$
h_{\mu} \delta(x-k)=(k z)^{\frac{1}{2}} J_{\mu}(k z)=F(z) \text {. }
$$

Now

$$
F\left(\lambda_{n}\right)=\left(k \lambda_{n}\right)^{\frac{1}{2}} J_{\mu}\left(k \lambda_{n}\right), \quad n=1,2,3, \ldots
$$

$$
\begin{aligned}
<\frac{2}{b^{2}} \sum_{n=1}^{N}\left(\frac{x}{\lambda_{n}}\right)^{\frac{1}{2}} & \frac{J_{\mu}\left(x \lambda_{n}\right)}{J_{\mu+1}^{2}\left(b \lambda_{n}\right)}\left(k \lambda_{n}\right)^{\frac{1}{2}} J_{\mu}\left(k \lambda_{n}\right), \phi(x)> \\
& =\frac{2}{b^{2}} \sum_{n=1}^{N} \frac{J_{\mu}\left(k \lambda_{n}\right)}{\left(\lambda_{n}\right)^{\frac{1}{2}} J_{\mu+1}^{2}\left(b \lambda_{n}\right)}(k)^{\frac{1}{2}} \int_{0}^{f b}\left(x \lambda_{n}\right)^{\frac{1}{2}} J_{\mu}\left(x \lambda_{n}\right) \phi(x) d x \\
& =\frac{2}{b^{2}} \sum_{n=1}^{N} \frac{J_{\mu}\left(k \lambda_{n}\right)}{\left(\lambda_{n}\right)^{\frac{1}{2}} J_{\mu}^{2}\left(b \lambda_{n}\right)}(k)^{\frac{1}{2}}{ }_{\phi}\left(\lambda_{n}\right) \\
& =\phi(k) \\
& =<\delta(x-k), \phi(x)>.
\end{aligned}
$$

This also yields

$$
\delta(x-k)=\lim _{N \rightarrow \infty} \frac{2}{b^{2}} \sum_{n=1}^{N} \sqrt{k x} \frac{J_{\mu}\left(k_{n}\right) J_{\mu}\left(x_{n}\right)}{J_{\mu}^{2}\left(b_{\lambda_{n}}\right)}
$$

in the sense of convergence in $\mathrm{B}_{\mu, \mathrm{b}}^{\prime}$.

ACKNOWLEDGEMENT: This work was supported by Grant No. 1402/28 from Science Center, King Sauci University, Saudi Arabia.

\section{REFERENCES}

1. Zemanian, A. H.. A distributional Hankel transform, Siam J. App. Math., vol. 14, No. 3, 1966, pp. 561-576.

2. Zemanian, A. H.. The Hankel transformation of certain distributions of rapid growth, Siam J. Appl. Math., vol. 14, No. 4, 1966.

3. Schwartz, L.. Theorie des distributions, Herman, Paris, 1978.

4. Zemanian, A. H.. Generalized integral transformations, Interscience Publishers, New York, 1968.

5. Zemanian, A. H.. Distribution theory and transform analysis, McGraw-Hill, New York, 1965.

6. Sneddon, I. N.. The use of integral transforms, McGraw-Hill Book Company, 1972.

7. Watson, G. N.. Theory of Bessel functions, 2nd ed., Cambridge University Press, 1958. 
8. Schwartz, L.. Mathematics for the physical sciences, Addison Wesley Publishing Company.

9. Carmichael, R. D.. Analytic representation of the distributional finite Fourier transform, Siam J. Math Anal. $\underline{5}(\underline{5}), 1974,737-761$.

10. Warmbrod, G. K.. The distributional finite Fourier transform, Siam J. Appl. Math, $\underline{17}(\underline{5}), 1969,930-956$. 


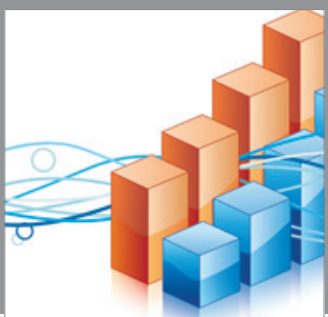

Advances in

Operations Research

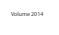

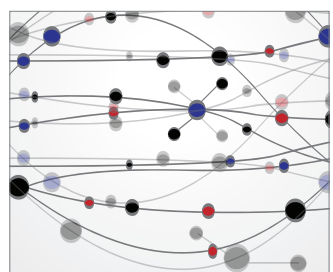

\section{The Scientific} World Journal
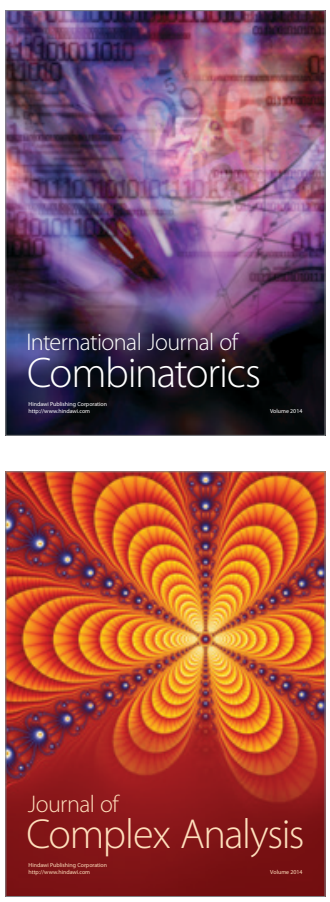

International Journal of

Mathematics and

Mathematical

Sciences
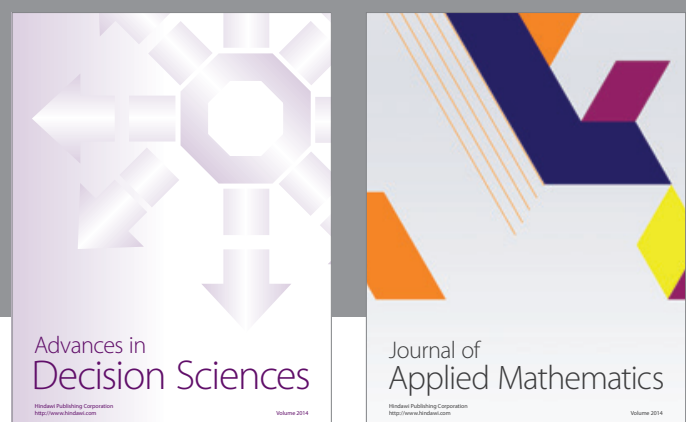

Journal of

Applied Mathematics
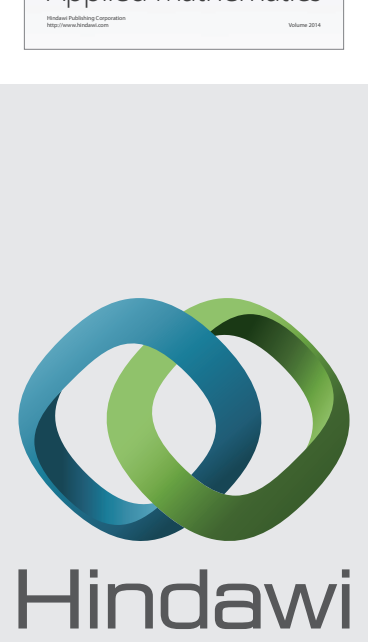

Submit your manuscripts at http://www.hindawi.com
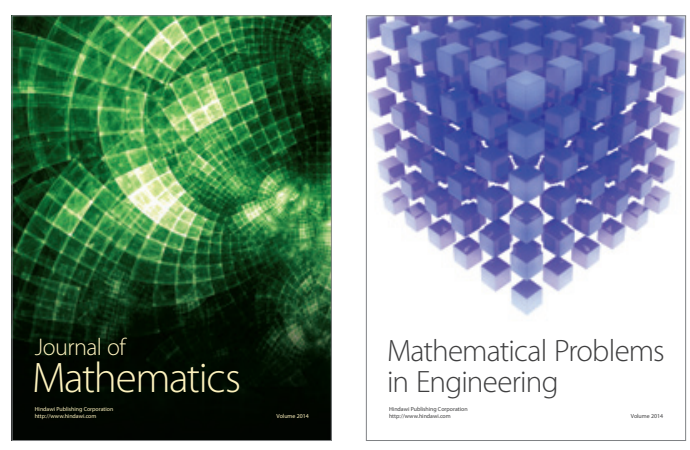

Mathematical Problems in Engineering
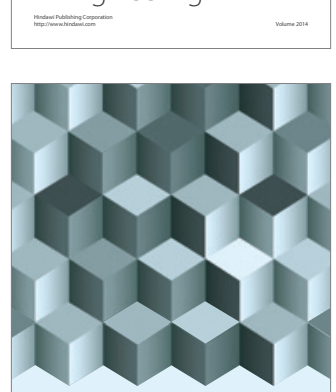

Journal of

Function Spaces
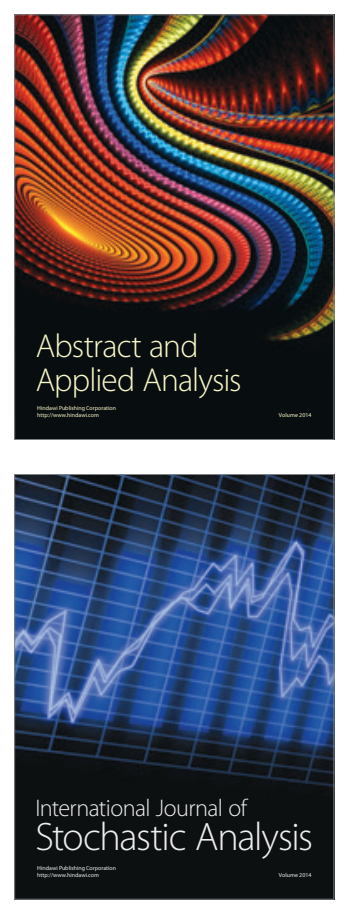

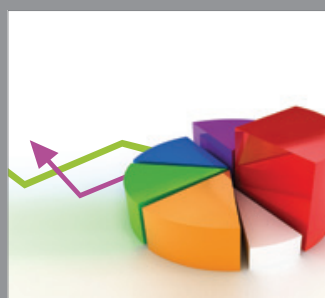

ournal of

Probability and Statistics

Promensencen
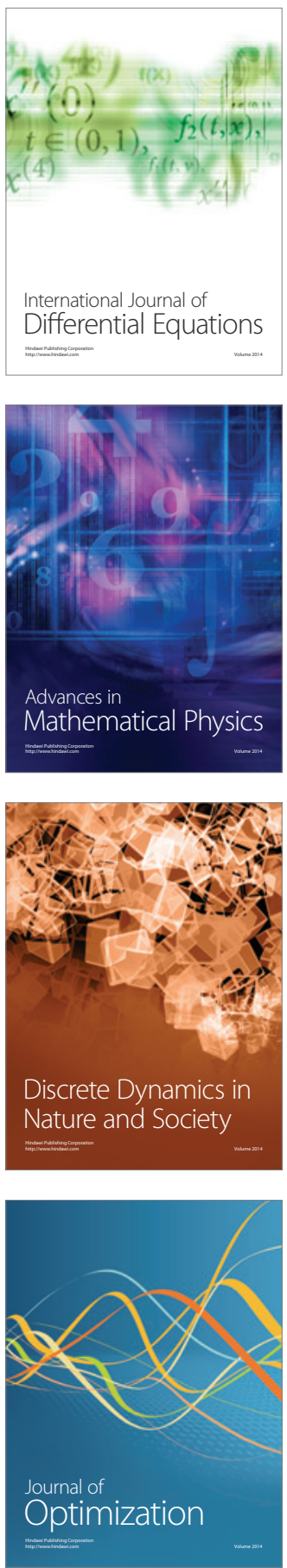